estudios del habitat $\left.\right|_{\text {FAU: }}=$
Estudios del Hábitat

ISSN: 2422-6483

aefau@fau.unlp.edu.ar

Universidad Nacional de La Plata

Argentina

\title{
Políticas urbanas, conflicto y territorio: complejidades de la acción público-privada en la producción del espacio
}

Arqueros Mejica*, María Soledad

Políticas urbanas, conflicto y territorio: complejidades de la acción público-privada en la producción del espacio

Estudios del Hábitat, vol. 18, núm. 1, 2020

Universidad Nacional de La Plata, Argentina

Atribución no comercial compartir igual (CC BY-NC-SA) 4.0 
Políticas urbanas, conflicto y territorio: complejidades de la acción público-privada en la producción del espacio

\author{
María Soledad Arqueros Mejica* \\ Consejo Nacional de Ciencia y Técnica. Instituto de \\ Investigaciones Gino Germani. Facultad de Ciencias \\ Sociales. Universidad de Buenos Aires, Argentina \\ soledad.arqueros@gmail.com
}

Recepción: 23 Octubre 2019

Aprobación: 15 Abril 2020

Publicación: 30 Junio 2020

Recepción: 23 Octubre 2019

Aprobación: 15 Abril 2020

Publicación: 30 Junio 2020

\section{Resumen:}

Este artículo focaliza la atención en el vínculo entre las transformaciones espaciales y los procesos de neoliberalización en la Ciudad de Buenos Aires. A partir del caso del Polo Farmacéutico del barrio de Villa Soldati, indaga en las relaciones que establecieron las organizaciones empresariales, estatales y sociales en el marco del proyecto, los conflictos y disputas que tuvieron lugar en el territorio y sus efectos en la política de desarrollo. El análisis muestra las complejidades que atraviesa la acción público-privada en la producción del espacio, poniendo de relieve el papel que juegan las condiciones de contexto, el territorio y las temporalidades de los distintos actores en esos procesos. Asimismo, evidencia las discusiones en torno a los sentidos, significados y condiciones materiales del desarrollo que tuvieron lugar en el marco del conflicto, dando cuenta de la capacidad de incidencia que tienen sectores ajenos a los intereses dominantes en la política urbana.

PAlabras ClAVE: entramados público-privados, políticas públicas, desarrollo urbano, conflictos de proximidad, productividad del conflicto social.

\section{ABstract:}

This paper focuses on the relationship between neoliberalization processes and territorial transformation in Buenos Aires city. Based on Villa Soldati's Pharmaceutical hub, I explore the relationships that established entrepreneurial, state and social organizations in this project, the conflicts and disputes that had place in the territory and its effects on development policy. The analysis shows the complexities that go through public-private action, highlighting the role played by context conditions, the territory and temporalities of the different actors in those processes. Furthermore, evidence the discussions about the senses, meanings and development material conditions that took place in the context of the conflict, showing the incidence capacity of sectors that doesn't have coincidence with dominant interests in urban policy.

KEYWORDS: public-private frameworks, public policies, urban development, proximity conflicts, social conflict productivity.

\section{INTRODUCCIÓN}

La conformación de polos productivos cobró centralidad a partir de la década de 1970 en el contexto de transformaciones del modo capitalista, constituyéndose en una de las manifestaciones de la globalización y de la competencia entre ciudades. Desde entonces, distintos gobiernos se apoyaron en este tipo de iniciativas, presentándolas como una solución para la rehabilitación de áreas obsoletas. Este fue el caso de la ciudad de Buenos Aires, que en la década del 2000 promovió la creación de una diversidad de polos en el área sur, aunque niguno logró completarse en su totalidad (Socoloff, 2013). Este artículo reflexiona sobre la relación entre las transformaciones espaciales y los procesos de neoliberalización (Brenner, et. al., 2013; Harvey, 2005) a partir del proyecto de emplazar un Polo Farmacéutico en el barrio de Villa Soldati de la Comuna 8 de la Ciudad de Buenos Aires, en terrenos que la Villa 20 reclamaba para avanzar en su proceso de reurbanización. Específicamente, se interesa en las complejidades que asumieron las relaciones entre las organizaciones empresariales, estatales y sociales en el marco de ese proyecto, los conflictos y disputas que 
tuvieron lugar en el territorio y sus efectos en la política de desarrollo de la Comuna 8. Esto permite contribuir a la caracterización del neoliberalismo realmente existente(Theodore, et. al., 2009) en la Ciudad de Buenos Aires e interrogar la estrategia de creación de polos y distritos económicos para desarrollar su zona sur ${ }^{1}$.

La Comuna 8 está localizada en el sur de la ciudad de Buenos Aires. Se caracteriza por tener bajas densidades; un tejido discontinuo y atravesado por barreras urbanas; un stock industrial en desuso y declive de la actividad económica; y por concentrar la mayor cantidad de villas y vivienda social de la ciudad ${ }^{2}$. Pero también presenta cierta disponibilidad de terrenos fiscales y una localización central en el Área Metropolitana de Buenos Aires (Figura 1). El proyecto de emplazar un Polo Farmacéutico se inserta en la política de desarrollo del sur, que en este periodo se orientó a la expansión del mercado en el área. En el caso analizado esto se observa en el surgimiento de una modalidad de desarrollo conducida por el mercado; un avance de los sectores empresariales en la definición de intervenciones de política urbana; y en la privatización de terrenos fiscales, que tenía como contracara la desposesión de los habitantes de Villa 20.

A pesar de ser promovido por empresarios y una cámara farmacéutica, de contar con el apoyo explícito del Jefe de Gobierno de la Ciudad y de algunas agencias del Gobierno Nacional, el proyecto del Polo Farmacéutico no logró materializarse por completo. El análisis de su trayectoria evidencia que los procesos de producción del espacio convocan a una diversidad de actores que toman decisiones y despliegan cursos de acción en base a dinámicas globales, nacionales y locales. Asimismo, pone de relieve que el contexto histórico y territorial (Rodríguez y Di Virgilio, 2011) en donde se despliega la acción individual y colectiva de esos grupos, incide en el curso y orientación de los procesos urbanos. Esto permite matizar miradas más mecánicas sobre las articulaciones entre actores empresariales (o capitalistas) y estatales en la producción de la ciudad neoliberal y ponderar la capacidad de agencia de grupos cuyas prioridades y requerimientos se diferencian de los dominantes, así como sus efectos en el curso de la política (Melé, 2016). A su vez, destaca el carácter conflictivo, contradictorio y, muchas veces, improvisado de las políticas urbanas.

En este sentido, el presente artículo reflexiona sobre los procesos de neoliberalización desde una perspectiva teórica que, aun con tensiones, reconoce el peso que tienen los procesos estructurales propios del desarrollo del capitalismo, pero también los cursos de acción que despliegan los actores sociales en la configuración del espacio urbano (García y Rofman, 2013). A partir de autores como Sanfelici (2017), Martin (2011), Rodríguez (2008), Pírez (1995) y Harvey (1989), la urbanización es visualizada como un producto y un proceso de circulación secundaria del capital y, al mismo tiempo, un medio y recurso para la acción política, en cuyo marco grupos sociales disímiles disputan las condiciones de producción, consumo y goce de la ciudad.

La política pública se revela en este contexto como una poderosa matriz analítica. En base a diversos autores (Laucomes y Le Galés, 2012; Rodríguez y Di Virgilio, 2011; Oszlak y O’ Donnell, 2008 [1976]), la cuestión del Polo Farmacéutico es conceptualizada aquí como un proceso político, tejido en torno a un asunto problemático, que convoca a actores con distintas concepciones e intereses y que desarrollan cursos de acción en el marco de determinadas relaciones de poder $^{3}$. En esta línea, como señala Marques (2016), la influencia de los capitalistas (en este caso los empresarios farmacéuticos) en la formulación de políticas está estrechamente ligada al uso de recursos de poder, a la adopción de estrategias políticas en relación con diversos actores, y a las instituciones estatales involucradas en la producción de esas políticas. En el caso analizado, parafraseando a Harvey (2007), podemos decir que los empresarios farmacéuticos lucharon por hacer su propia geografía histórica, pero lo hicieron en condiciones históricas y geográficas que no fueron de su elección individual. En particular, el territorio se constituyó en un ámbito en donde diferentes actores disputaron y negociaron el sentido, orientación y las condiciones materiales del desarrollo, protagonizando una serie de conflictos (Melé, 2016) que incidieron y recrearon la política urbana.

La investigación se sostuvo en una metodología de carácter cualitativo. Por un lado, se realizaron entrevistas a líderes sindicales de los laboratorios involucrados, a los estudios de arquitectura y empresas constructoras que participaron del proyecto, y a funcionarios públicos y referentes de organizaciones territoriales que avalaron o se opusieron a su ejecución. Por el otro lado, se retomaron declaraciones 
públicas de los empresarios farmacéuticos en medios de prensa nacionales y específicos del sector; las realizadas en audiencias y actos públicos; y documentos y solicitadas emitidos por la cámara farmacéutica COOPERALA. Asimismo, se recuperaron las leyes, versiones taquigráficas de sesiones parlamentarias y expedientes legislativos y judiciales atinentes al proyecto. Esas fuentes fueron trabajadas mediante la técnica de análisis documental, desde una perspectiva que las reconoce como hechos sociales (Atkinson \& Coffey, 2011), que además de proveer información, dan cuenta del modo en que las organizaciones representan sus realidades, de los públicos a los que se dirigen, los repertorios de acción de promueven y de las miradas de futuro que estimulan.

El artículo se divide en cinco apartados, que recorren la trayectoria del Polo Farmacéutico (Oszlak y O 'Donnell [1976]). El primero reconstruye brevemente los intereses y motivaciones de sus promotores. El segundo se concentra en las controversias que esta iniciativa generó en la legislatura, entre las agencias estatales, los habitantes y organizaciones del área. El tercero focaliza la atención en los conflictos de proximidad que provocó el proyecto y su productividad (Melé, 2016). Muestra también cómo fueron variando las posiciones y relaciones entre esos actores, los cursos de acción que desplegaron en el marco del conflicto y sus efectos en la política. Los últimos dos apartados se detienen en el modo en que los cambios en las coyunturas políticas y económicas (Cochrane, 1999) afectaron las formas de coordinación entre el sector público y el sector privado, y entre los laboratorios que promovían la iniciativa. Hacia el final del artículo, se reflexiona sobre lo trabajado.

\section{QuiÉnes, cómo Y PORQuÉ: LOS PROMOTORES DEL Polo}

El proyecto del Polo Farmacéutico fue impulsado por la cámara de laboratorios farmacéuticos nacionales COOPERALA en un contexto de pérdida de competitividad del sector ${ }^{4}$. Asimismo, en el año 2002, la Administración Nacional de Medicamentos, Alimentos y Tecnología Médica (ANMAT), había adoptado una serie de regulaciones y protocolos que obligaban a los laboratorios a ampliar y readecuar sus plantas en un plazo de 5 años. Luego de recorrer diversos municipios del Área Metropolitana de Buenos Aires, COOPERALA llegó a un acuerdo con la Corporación Buenos Aires Sur ${ }^{5}$ (CBAS) para emplazar el Polo en terrenos del barrio de Villa Soldati de la Comuna 8, aledaños a la Villa $20^{6}$ y a diferentes conjuntos de vivienda social (ver Figura 1).

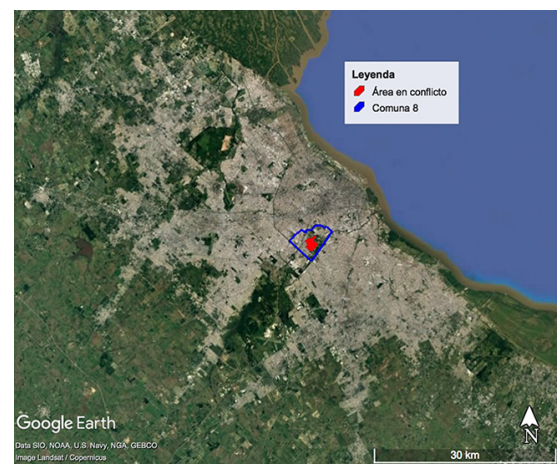

FIGURA 1

Localización de la Comuna 8 y del área en conflicto en el Área Metropolitana de Buenos Aires

ELABORACIÓN PROPIA SOBRE UNA IMAGEN SATELITAL DE GOOGLE EARTH (2020)

La propuesta fue recibida con entusiasmo por el gobierno local, que en el contexto nacional de salida de la convertibilidad y crecimiento de la economía del periodo neodesarrollista ${ }^{7}$, veía en la industria de la construcción y la renta productiva la posibilidad implementar proyectos de "alto impacto sobre el territorio" (GCBA, 2004a). El Polo Farmacéutico, señalaba Ibarra, permitiría “[...] recuperar la historia y la memoria 
productiva de la zona sur" y daba cuenta de "la intervención y voluntad del Estado en desarrollar [esa] zona" (Anibal Ibarra en Redacción Clarín, 2004). La industria farmacéutica tenía un peso creciente en la ciudad, era una de las que presentaba mayor cantidad de personal asalariado y mejores perspectivas de crecimiento. Además, en este periodo, se construyó en el área un centro de logística farmacéutica en donde participan laboratorios que también estaban involucrados en el proyecto del Polo (por ejemplo, Montepellier, Bagó y Omicrom).

"Se trata de una decisión estratégica para desarrollar una industria con potencial" señalaba el Jefe de Gobierno Aníbal Ibarra durante un acto con empresarios del sector (Redacción La Razón, 2004). A lo que Enrique Rodríguez, por entonces presidente de la CBAS agregaba, "El polo Farmacéutico [...] va a permitir ampliar y modernizar la exportación, lo que generará beneficios para Buenos Aires. Además, revalorizará la zona y generará fuentes de trabajo para los vecinos" (Redacción La Razón, 2004). El emprendimiento aparecía así como una oportunidad para retener la industria farmacéutica en la jurisdicción de la ciudad, en un contexto marcado por la recuperación del Producto Bruto Geográfico (que se había contraído en la crisis de 2001) y por el interés de reposicionarla en el mercado internacional (Arqueros Mejica, 2018a).

El proyecto también generó entusiasmo en algunas reparticiones del Estado Nacional. En particular, recibió el apoyo de la Agencia de Promoción Científica y Tecnológica de la Nación (ANPCyT) y posteriormente del Ministerio de Ciencia y Técnica de la Nación, ambos conducidos por el Doctor en Ciencias Químicas Lino Barañao, que apostaba a través de políticas activas a la expansión del sector. Desde esta dependencia se presumía que el Polo contribuiría a la investigación científica, el desarrollo y la innovación tecnológica (I+D) en el área farmacéutica y, en particular, biotecnológica (Juncal et. al., 2013). Así, hacia el año 2006, se conformó una asociación ad hoc con COOPERALA, el Consejo Nacional de Investigaciones Científicas y Técnicas (CONICET), la Facultad de Farmacia y Bioquímica de la Universidad de Buenos Aires, la Universidad Abierta Interamericana, la Fundación Pablo Cassará -ligada al laboratorio homónimo-, la CBAS, el Ministerio de Salud de la Nación y la Administración Nacional de Laboratorios para desarrollar un conjunto de acciones, entre las que se incluía el proyecto en cuestión (Arqueros Mejica, 2018a).

\section{Controversias}

\section{- El debate en la Legislatura: presiones, apoyos y disidencias}

En el año 2004, el ex Jefe de Gobierno Aníbal Ibarra envió a la legislatura un proyecto de ley de rezonificación de terrenos para habilitar la construcción del Polo. Se esperaba iniciar las obras en 2005 y que estuviera en pleno funcionamiento en 2008. Entre las tierras dispuestas para su emplazamiento se incluía un predio que el Instituto de la Vivienda de la Ciudad (IVC), que es el ámbito de aplicación de la política habitacional, había afectado apenas unos meses antes a la reurbanización de la Villa 20 (Figura 2). 


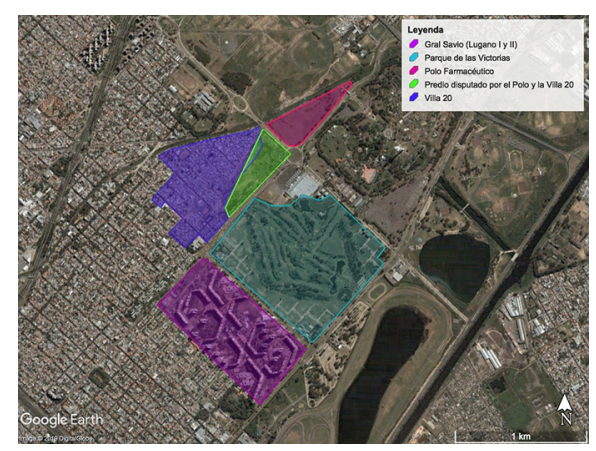

FIGURA 2

\section{EMPLAZAMIENTO DEL POLO FARMACÉUTICO Y BARRIOS ALEDAÑOS \\ ELABORACIÓN PROPIA SOBRE UNA IMAGEN SATELITAL DE GOOGLE EARTH (2004)}

El proyecto tuvo dos dictámenes en la Comisión de Planeamiento, que daban cuenta de los distintos modos de entender el desarrollo ${ }^{8}$. El de mayoría partía de la concepción de “desequilibrios territoriales”, en donde la revitalización urbana del sur aparecía estrechamente vinculada al crecimiento económico y la dinamización productiva, bajo el supuesto de que esto promovería la reconversión territorial y generaría fuentes de empleo. En esta línea, una de sus autoras señalaba que,

El proyecto del Polo [Farmacéutico] impulsará el desarrollo del área sur de la ciudad, incorporando nuevas actividades económicas y de investigación. Fomentará iniciativas privadas de inversión y revalorizará definitivamente la zona de su emplazamiento, generando actividades directas e indirectas [ex legisladora Caruso, en Legislatura Porteña (2004a: 156)].

Esta postura sostenía que la participación del sector privado era uno de los pilares de la política de desarrollo. En el marco de estas definiciones, el fortalecimiento y retención de la industria farmacéutica en la ciudad y el desarrollo de la zona sur aparecían estrechamente entrelazados.

Un grupo de legisladores presentó un dictamen de minoría, que partía de una concepción más cercana a la idea de "desigualdades", que disputaba sentidos y orientaciones de política con la de "desequilibrios". Allí se solicitó el archivo del proyecto, argumentando que el Polo crearía una barrera urbana, acentuando la segregación y desintegración. Esto quedó expresado en el discurso de uno de los legisladores, que sostuvo que,

[...] en este proyecto, se está planteando un modelo de ciudad, un modelo productivo, un modelo que va a seguir profundizando las desigualdades y la polarización de esta Ciudad que atenta [...] contra cualquier instancia legítima y democrática de desarrollo y de estrategia de construcción hacia un hábitat más democrático [...] [ex legislador Molina, en Legislatura Porteña (2004a: 160)].

Este grupo puso en discusión la mercantilización y venta de terrenos públicos al sector privado, que aparecían ligados a la discusión de las formas de desarrollo, “[...] nos imaginamos todas estas enormes e irrecuperables tierras de reserva con que cuenta la Ciudad para desarrollar proyectos de utilidad pública de alcance masivo, que pueden orientarse a un destino de desarrollo y de modelo de ciudad distinta" [ex legislador Molina, en Legislatura Porteña (2004a: 159)]. En particular, se cuestionó la apropiación y uso de los terrenos dispuestos por el IVC para la radicación de la Villa 20, así como la promoción de esta iniciativa en un contexto en que la construcción de un Hospital General de Agudos - que era un reclamo histórico de la población del área - era desestimada.

De esta manera, el debate legislativo dejaba ver distintos puntos de vista a la hora de pensar y promover el desarrollo, cuestión que, como se verá más adelante, estaba en sintonía con lo que ocurría en el ámbito del ejecutivo local y en el territorio. Más allá de esas diferencias, la ley de rezonificación de terrenos resultó aprobada en primera lectura constituyendo un primer avance del proyecto. 


\section{- LAS CONTROVERSIAS EN EL EJECUTIVO LOCAL}

El avance del proyecto en la Legislatura dio lugar a una serie de controversias sobre la orientación e implicancias del "desarrollo", que involucraron a actores de lo más diversos, quienes interpretaron y procesaron la iniciativa a la luz de sus propios intereses y prioridades. Estas discusiones quedaron expresadas en la audiencia pública celebrada en diciembre de 2004 (Legislatura Porteña, 2004b) en el marco del procedimiento de la ley, en donde funcionarios de organismos estatales, habitantes y organizaciones territoriales, y empresarios farmacéuticos explicaron y argumentaron sus respectivas posturas.

En el ámbito del ejecutivo local la iniciativa enfrentó a la CBAS y el IVC, que en ese periodo tenían una relación de conflicto y competían por la apropiación, uso y destino de los terrenos de Villa Soldati (Arqueros Mejica, 2018b). La sanción en primera lectura de la ley, produjo malestar en el IVC, que señaló que hacía mucho tiempo venía 'peleando y trabajando por el traspaso de los terrenos'. Que para ello habían mantenido 'innumerables reuniones' con funcionarios del gobierno nacional -que tenía el dominio de las tierras- 'para tratar de encontrar soluciones' que les permitieran 'tener en la mano esos terrenos y empezar a trabajar en el proyecto' de reurbanización de la Villa 20.

La CBAS contraargumentó que para construir en ese predio era necesario realizar un estudio de suelo y proveer infraestructura de servicios, aspectos que demorarían el proyecto de reurbanización, por lo que era preferible destinarlo al Polo. Sostuvo que en caso de no efectivizarse el Polo, 'la industria se va de la ciudad' y que esto significaría la pérdida de uno de los sectores 'con mayor valor agregado' y que 'tiene capacidad exportable'. Agregó que desde ese ámbito se buscaba 'tener un anclaje de desarrollo con empleo', que si 'se pierde la industria, se pierde trabajo' y que 'resolver la pobreza sin trabajo y sin empleo, no es resolverla'.

Finalmente, para destrabar el conflicto, la CBAS, tomando nota de que la reurbanización de la Villa 20 era 'una reivindicación histórica', propuso 'despojarse' de 4 hectáreas localizadas en el Parque de las Victorias, que eran 'lo más valioso' que tenía, a cambio del predio. El IVC aceptó la oferta, argumentando que esto le permitía disponer 'en forma inmediata' de terrenos que serían destinados a la reurbanización de Villa 20.

\section{- LAS CONDICIONES MATERIALES Y SENTIDOS DEL "PROGRESO"}

Esa resolución del problema generó un nuevo conflicto, esta vez entre las organizaciones del Estado y las de base territorial, que tenían reservas con respecto a la instalación del Polo, las formas de desarrollo que se promovían y el modo en que el IVC había manejado el tema. En este sentido, como señalan Oszlak y O’Donnell (2008 [1976]), la toma de posición del Estado frente a una cuestión socialmente problematizada, tiende a generar respuestas de otros actores y una trama de interacciones que definen y reencuadran el proceso. La negociación la CBAS y el IVC para el emplazamiento del Polo, generó condiciones para la emergencia de un debate sobre las condiciones materiales, los sentidos y alcances del "progreso" -o desarrollo-, convirtiéndolo en un tema público, aspecto que remite a la productividad política de los conflictos urbanos (Melé, 2016).

En este contexto, el debate se fue desplazando desde la cuestión puntual del Polo Farmacéutico a un campo problemático más amplio. El uso y apropiación de la tierra aparecía como un elemento que generaba confrontaciones de mayor envergadura, en donde lo que se estaba discutiendo eran las condiciones materiales y los significados del desarrollo. En este sentido, parafraseando a Kowarick (1996), podemos decir que el proyecto del Polo dio lugar a un proceso de producción de experiencia en donde los diversos actores involucrados interpretaron y elaboraron discursos, que fueron significando y dando sentido a esa materialidad objetiva. Los empresarios farmacéuticos entendían el progreso a partir del binomio inversióntrabajo, alentando un proceso que los pobladores interpretaban como un despojo de sus derechos en torno a la calidad de vida y el acceso a un hábitat digno. 
En este sentido, los habitantes de la Villa 20 cuestionaron que no se los hubiera incorporado al 'debate del Polo', señalaron que estaban en 'contra de que se hagan las cosas entre gallos y medianoches' y reclamaron que se 'elabore una nueva ley' con su participación. Argumentaron que estas tierras pertenecían a los habitantes de Villa 20, que se las habían ganado a través de 'largos años de luchas y ordenanzas, de leyes y decretos', poniendo de este modo el reclamo en el ámbito de lo jurídico.

COOPERALA y los laboratorios señalaron que no entendían por qué 'alguien podía oponerse' a este proyecto, que buscaba 'aportar al crecimiento' y que daría 'empleos', 'ingresando al mercado de trabajo a los excluidos' y produciendo un 'efecto multiplicador en la zona'. Añadieron que en 'nombre de la globalización' se había descuidado el 'capital social' y que las condiciones de 'desigualdad y desocupación' que afectaban a 'muchos compatriotas' solo se podían solucionar si 'hay empresas privadas que produzcan', 'paguen sus impuestos' y 'aumenten las exportaciones y el producto'.

Desde las organizaciones señalaron que debía 'quedar bien claro cuál es el desarrollo', que ellos tenían 'derechos', y que si 'viene el Polo Tecnológico' que también 'vengan los servicios básicos para la gente de la villa'. Insistieron en que 'la tierra' era un 'bien preciado' que estaban 'perdiendo' en 'manos del progreso' y les dijeron a los 'señores que han estudiado', 'que se han capacitado' y 'ven sus futuros', que ellos también querían que 'vean' sus 'futuros'. Sostuvieron que su reclamo era una 'ciudad para todos' y que si la 'ciencia y la técnica' estaban 'al servicio del hombre', 'en absoluto' iban a estar 'en contra del progreso'.

\section{Conflicto. NuEvos INTERCAMBios, REPOSICIONAMIENTOS Y ACCIÓN POLÍTICA}

Estos debates y definiciones interpelaban al Estado en su función central de garantizar las condiciones que permiten la reproducción de la urbanización capitalista. En este marco, como señala Kowarick (1996), el Estado "se politiza" tornándose el centro de presiones y reivindicaciones, transformándose en el núcleo de los conflictos. Esto fue visible en el caso analizado, en donde los distintos actores involucrados desplegaron una serie de acciones que incidieron en el curso de la política.

Luego de la audiencia pública celebrada en el año 2004 continuaron produciéndose intercambios entre los sectores estatales y no estatales, se sumaron a la discusión nuevos grupos y se produjeron reposicionamientos entre los que estaban involucrados. En los primeros meses del año 2005 la Corporación impulsó negociaciones entre las agencias y sectores que se oponían al Polo y presionó a la Legislatura para que tratara en segunda lectura el proyecto de rezonificación de los terrenos.

En este marco, promovió la firma de un Acta de acuerdo entre algunas agencias estatales y referentes de la Villa 20, en donde se comprometieron a trabajar conjuntamente en el proyecto de urbanización de la villa y cedieron los terrenos requeridos para el Polo, a cambio de los ofrecidos en el Parque de las Victorias (Figura 2). El trato era que estos referentes podrían participar a través de sus cooperativas en la construcción de las viviendas de Villa 20. Esto modificó la "contracoalición" conformada en torno al proyecto, provocando redefiniciones y reorientaciones en el proceso. Concretamente, la firma del acuerdo produjo una división entre las organizaciones territoriales, que en el nuevo contexto y a partir sus propios objetivos fueron variando su posición.

En este sentido, como señala Pírez (1995: 10), “[...] los actores no actúan sus determinaciones estructurales, sino que ellas operan mediadas por la comprensión que realizan de sus finalidades y del contexto en el cual las buscan". Los términos del Acta abrieron paso a un repertorio de interpretaciones entre las organizaciones. Un grupo aceptaba el Polo y el canje de terrenos acordado entre la CBAS y el IVC, a cambio de participar en la construcción de viviendas, que era interpretada como una posibilidad para acceder a trabajo y una mejor calidad de vida, al tiempo que otorgaba beneficios particulares a sus propias cooperativas. El otro grupo, en cambio, se oponía de manera tajante al emplazamiento del Polo y a la construcción de viviendas en el Parque de las Victorias, alegando que esto generaría un impacto nocivo a nivel ambiental y afectaría la calidad de vida en el área. 
Esta división se expresó también entre los legisladores (Legislatura Porteña, 2005a y 2005b) quince días más tarde, cuando se propuso tratar 'sobre tablas' la segunda lectura del proyecto de ley de rezonificación de terrenos. Esto provocó fuertes cruces entre los diputados de la ciudad y entre las organizaciones, al punto de que por un momento "parecía inminente la toma del recinto" (Redacción Noticias Urbanas, 2005). La sesión debió ser suspendida y el proyecto del Polo volvió a quedar trabado.

\section{- Nuevos actores, Protesta callejera y REClamo Judicial}

Tal como señala Melé (2016) los conflictos producen formas positivas de socialización, aportando a la conformación de alianzas, asociaciones y redes. La propuesta de construir las viviendas en el parque implicó la re-territorialización del conflicto, interpelando a los habitantes del conjunto habitacional Gral. Savio1 (en adelante, Lugano I y II) (Figura 2), que hasta entonces habían tenido una actitud distante con la población de la villa. A estos grupos se sumó también un conjunto de vecinos que reclamaba la construcción del Hospital General de Agudos en el área. A partir de estas rearticulaciones se conformó la Coordinadora Multisectorial Sudoeste $1^{10}$.

La Multisectorial se opuso al Polo y a la construcción de viviendas en el Parque de las Victorias, en palabras de uno de sus integrantes "[...] nosotros no aceptábamos la negociación, queríamos la urbanización y el hospital" [Sebastián, integrante de la Multisectorial Sudoeste, entrevista realizada en enero de 2017]. En ese marco, organizaron asambleas públicas, movilizaciones, cortes de calle y volanteadas, explicando que “[...] era mentira que [el Polo] iba a generar empleo", que en cambio iba a producir "problemas ambientales graves" y que el "[...] tema es si esto es realmente progreso para la comuna" (el resaltado es mío) [Sebastián, integrante de la Multisectorial Sudoeste]. Esto les permitió lograr visibilidad, deslegitimar el Acta de Acuerdo firmada y frenar el tratamiento definitivo de la ley.

Desde este espacio percibían que se estaban enfrentando a una industria "especialmente poderosa”, que planteaba un "desafío más complejo" [Sebastián, integrante de la Multisectorial Sudoeste], por lo que se decidió combinar la protesta en el espacio público con el reclamo judicial. En junio de 2005 se presentó un recurso de amparo solicitando una medida cautelar, que fue concedida por el juez a cargo de la causa. En base a Sabatini (1999), podemos señalar que la intervención del poder judicial produjo un cambio en las relaciones de fuerza, ya que obstaculizó transitoriamente el proyecto del Polo, impidió la construcción de viviendas en el Parque de las Victorias y priorizó las necesidades y reclamos de los habitantes del área ${ }^{11}$.

El disparador de la acción fue el llamado a licitación para la construcción de cuatro torres en terrenos aledaños al conjunto Lugano I y II. La medida cautelar emitida por el juez impidió la realización de construcciones nuevas en el Parque de las Victorias y en el predio del Polo, alegando la ausencia de estudios de impacto ambiental que evidencien los efectos de estos emprendimientos en la zona (Poder Judicial de la Ciudad Autónoma de Buenos Aires, 2005).

La medida tuvo un efecto directo en el orden normativo, lo que pone de relieve la productividad jurídica del conflicto (Cosacov, 2014). Específicamente, en agosto de 2005, la Legislatura aprobó con disidencias la ley 1.768 del Polo Farmacéutico ${ }^{12}$; y por unanimidad las leyes 1.769 de construcción de un Hospital General de Agudos en el área y 1.770 de reurbanización participativa de Villa 20. El Polo quedaría emplazado en un área más acotada que la original, prescindiendo de los terrenos que reclamaban los habitantes de Villa 20 (Figura 2). Aunque desde la Multisectorial Sudoeste esto fue visualizado como "[...] un acuerdo legislativo, que no era un acuerdo con los vecinos [....]", también se interpretó como una "victoria” porque “[...] los vecinos habían logrado la urbanización y el hospital” [Sebastián, integrante de la Multisectorial Sudoeste], aspecto que pone de relieve la capacidad -parcial- de incidencia que tienen las 'contracoaliciones' en las politicas urbanas. 


\section{ASINCRONÍAS}

Ahora bien, los proyectos como el Polo Farmacéutico, implican la coordinación de actores -empresarios y organizaciones estatales- que tienen distintas racionalidades y que despliegan sus prácticas en coyunturas políticas dinámicas. La racionalidad económica de los laboratorios no necesariamente coincidió con la racionalidad burocrática de las organizaciones estatales, en particular en un contexto marcado por cierta inestabilidad política, re-jerarquizaciones en la agenda estatal y reconfiguraciones en sus agencias. Esto trae a escena una dimensión central de estas formas de articulación: el tiempo, o más específicamente la temporalidad de la "agenda del que invierte" y su relación con la "agenda pública".

Como se señaló con anterioridad, el proyecto del Polo se gestó, en parte, por la necesidad de adaptar las plantas industriales a las regulaciones de la ANMAT. La rezonificación de los terrenos para emplazar el emprendimiento se aprobó de manera definitiva en 2005. Ese año se inició un proceso que decantó en el juicio político y destitución del Jefe de Gobierno Aníbal Ibarra, que había sido uno de sus principales impulsores. Esto retrasó los tiempos del proyecto: recién en 2006 se adjudicó la licitación para su construcción; y la escrituración de terrenos tuvo lugar a mediados de 2007, justo cuando se vencía el plazo otorgado por la ANMAT.

En diciembre de ese año, Mauricio Macri asumió la Jefatura de Gobierno. La nueva gestión concentró su atención en otros proyectos y zonas de la ciudad, como por ejemplo el Polo Tecnológico de Parque Patricios. ${ }^{13}$ A su vez, se modificó el perfil y papel de la Corporación Buenos Aires Sur. Estos cambios en los criterios de intervención, redefiniciones en las prioridades de política urbana y burocracias públicas, incidieron en los tiempos de ejecución del proyecto, que estuvo paralizado durante el primer año de esta gestión. Recién en 2009, por pedido de los propios laboratorios, el Centro de Atención al Inversor ${ }^{14}$ (CAI) comenzó a desarrollar una serie de acciones con el fin de acelerar su ejecución.

Para comenzar con las obras, el Polo debía contar con un certificado de impacto ambiental, que obtuvo en el año 2010. Antes de comenzar con la construcción de las plantas, era necesario resolver el tendido de infraestructura de servicios. Esto implicaba realizar una serie de gestiones con las empresas privadas de electricidad y gas natural -Edesur y Metrogas- y la empresa pública de agua y cloacas -AySA-, que aprobaron la construcción de las redes en el año 2012. Sobre este aspecto, una de las personas a cargo del proyecto señalaba que "fue difícil", "fue engorroso" el "tramiterío" con "los distintos organismos" [Carolina, arquitecta de la empresa a cargo del proyecto, entrevista realizada en septiembre de 2017].

Este derrotero muestra que las relaciones que establecen el sector público y el sector privado están atravesadas por una serie de complejidades (Rougieur, 2013; Beltrán, 2011; Dossi et. al., 2011) que permiten cuestionar el carácter unívoco que le asignan otras lecturas. Da cuenta, en cambio, que la coordinación entre este tipo de actores está lejos de constituir un proceso lineal y automático, en particular en contextos políticos dinámicos. En esta línea, el proceso del Polo abre interrogantes sobre las condiciones de posibilidad de los proyectos impulsados en el marco de entramados público-privados, que se profundizan al analizar las relaciones que se establecen al interior del "sector privado".

\section{El PROYECTO INCONCLUSO: CUANDO LOS LÍMITES PROVIENEN DEL PROPIO SECTOR PRIVADO}

Tal como señala Beltrán (2011), los empresarios tampoco constituyen un bloque homogéneo o monolítico, y sus intereses pueden ser divergentes e incluso contradictorios. Al interior de estos entramados pueden percibirse diferentes realidades, que tienen influencia en la toma de decisiones y en la posibilidad de generar acciones organizadas. También podemos decir que no "actúan" sus determinaciones estructurales, sino que organizan su acción en función de sus percepciones, intereses e interpretaciones (Pírez, 1995), en contextos políticos y económicos que los afectan. De este modo, aun reconociendo que su conducta y objetivos están 
mediados por su posición de clase, en cada coyuntura toman decisiones y despliegan cursos de acción a los que otorgan sentidos y significados particulares.

Aunque desde una mirada objetiva es discutible, los laboratorios nucleados en COOPERALA se consideraban a sí mismos pequeños y medianos empresarios. Como se señaló con anterioridad, el proyecto del Polo había nacido con el propósito de mejorar su competitividad a partir de la creación de economías de aglomeración. La salida de la convertibilidad generó un clima de negocios favorable para los laboratorios, que desde entonces buscaron incrementar la exportación. Este desenlace reforzó el objetivo general del proyecto, al que se sumó la necesidad de adaptar las plantas a las regulaciones de la ANMAT.

Sin embargo, entre las primeras definiciones del proyecto con la CBAS (2004) y la posibilidad de iniciar las obras (2012), pasaron ocho años. Durante ese periodo, cambiaron las necesidades, condiciones y recursos de las empresas que promovieron la creación del Polo. Muchas construyeron sus plantas en otras localizaciones y generaron estrategias alternativas para incrementar la exportación. De esta manera, cuando estaba todo listo para iniciar las obras, fue necesario redefinir los objetivos del emprendimiento, pero la situación y escala de cada laboratorio dificultó la posibilidad de llegar a un acuerdo.

En este sentido, el proyecto nucleaba a empresas como Química Montpellier del grupo Bagó, que era una de las más grandes a nivel local y que contaba con plantas en aproximadamente 20 países; y laboratorios pequeños, que tenían una producción más acotada y menor presencia a nivel internacional. Estas disparidades generaron disidencias internas con respecto a las finalidades del proyecto, así como criterios distintos sobre el modo de emprender acciones de producción y de investigación y desarrollo (Juncal et. al., 2013). Estas diferencias se profundizaron por la situación financiera de cada empresa, en un contexto de fuerte conflictividad entre los laboratorios y el Gobierno Nacional ${ }^{15}$.

Sin criterios unificados ni objetivos comunes y en una coyuntura política que fue evaluada como “desfavorable", los laboratorios dejaron en suspenso sus inversiones en el Polo. Aunque lograron comprar -en condiciones muy favorables- los terrenos para el emprendimiento, al 2019 solo construyó su planta Química Montpellier, que forma parte del poderoso grupo Bagó.

Esto pone de relieve que las empresas tienen una racionalidad limitada (Beltrán, 2011), toman decisiones en condiciones que no son de su propia elección, y que funcionan como un marco para evaluar su situación actual y extrapolarla a su futuro. Además, como se señaló anteriormente, los empresarios constituyen un grupo heterogéneo en cuanto a sus circunstancias particulares, intereses y lecturas de la realidad, aspecto que también dificulta la generación de consensos y, en algunos casos, su capacidad de organización. Estas complejidades repercutieron en la iniciativa del Polo, afectando la viabilidad del proyecto en su conjunto.

\section{REFLEXIONES FINALES}

Este artículo se propuso reflexionar sobre la relación entre las transformaciones espaciales y los procesos de neoliberalización de la Ciudad de Buenos Aires, a partir del conflicto suscitado en torno al Polo Farmacéutico del barrio de Villa Soldati. El foco estuvo puesto en las relaciones que establecieron las organizaciones estatales, empresariales y sociales en el marco del proyecto, las disputas que tuvieron lugar en el territorio y sus efectos en la política de desarrollo de la Comuna 8. Del análisis surgen algunos emergentes que interrogan la estrategia de creación de polos, y más recientemente, de distritos, para avanzar en el desarrollo del sur de la Ciudad de Buenos Aires.

Una primera observación es que la producción del proyecto neoliberal (Theodore et. al., 2009) en la Ciudad de Buenos Aires se apoya, en parte, en la expansión del mercado en áreas obsoletas y degradadas que, como la Comuna 8, históricamente fueron el lugar de residencia de sectores de la clase trabajadora. El 'desarrollo' aparece en este marco como un vehículo para el avance de los sectores empresariales en la definición de intervenciones de política urbana y para la privatización y mercantilización de terrenos fiscales, aspectos que ponen de relieve el componente extractivista (Svampa, 2011) que asumen estos procesos. 
Iluminar esta cuestión toma particular relevancia en el contexto actual, en que el gobierno de la ciudad fundamenta este tipo de intervenciones en un discurso anclado en la "mixturación social" del área a partir de este tipo de emprendimientos.

Pero el análisis evidencia también que los conflictos socio-territoriales y los vínculos entre las agencias del Estado y con los sectores empresariales, producen mediaciones en el alcance de las transformaciones territoriales que se promueven. En este sentido, la decisión de privatizar y transferir a los laboratorios terrenos públicos y un predio afectado a la urbanización de Villa 20, generó una serie de contradicciones y paradojas en la política de desarrollo, que incidieron en la génesis de un proceso de acción política en donde actores diversos disputaron las condiciones de producción, consumo y disfrute de la ciudad. En este marco, se puso en evidencia la centralidad del territorio, entendido como ámbito de la vida y de la política, en la disputa por el sentido, la orientación y las condiciones materiales y simbólicas del desarrollo.

Esto fue visible en las controversias y enfrentamientos que mantuvieron entre sí las distintas agencias del ejecutivo local y poderes del Estado, que pusieron en evidencia la convivencia de miradas diversas y variables sobre las maneras 'deseables' de avanzar en el desarrollo. A su vez, el territorio, se constituyó en un ámbito de socialización entre los diversos sectores -incluyendo a algunas agencias del Estado- que rechazaban el Polo. Las acciones desplegadas por esa red de organizaciones tuvieron efectos en la definición de la cuestión del desarrollo, en el orden jurídico-normativo y en la orientación de la política pública. Al mismo tiempo, logró revertir algunas decisiones que parecían inmodificables, como el emplazamiento del Polo en los terrenos dispuestos para la urbanización de Villa 20, evidenciando la capacidad de incidencia que tienen en la política urbana grupos y sectores cuyos intereses se diferencian de los dominantes.

El análisis desnaturaliza también los supuestos de armonía y sinergias que habitualmente se asigna en la utopía neoliberal a las relaciones que establecen el sector público y el sector privado. Las organizaciones estatales, por su particularidad, no pueden dedicarse sólo a garantizar la reproducción del capital, y aunque favorecieran a los empresarios, tienen rutinas, prácticas y procesos que delimitan temporalidades propias. Concretamente, la racionalidad económica de las empresas no siempre coincide con la racionalidad burocrática de las agencias estatales, generado desfasajes entre la agenda de los inversores y la agenda pública. El tiempo emerge así como una dimensión central a la hora de analizar las formas de coordinación entre ambos sectores y en las posibilidades que tienen de materializar los proyectos que promueven.

Por último, no pueden soslayarse las complejidades que asumen las relaciones entre los sectores empresariales y cómo afectan los proyectos que promueven. En este sentido, advertimos que los empresarios, aun desde una posición de clase, toman decisiones y despliegan acciones en función de sus objetivos y de las interpretaciones del contexto global, nacional y local en el que buscan realizarlos. En el caso analizado, el tamaño y recursos de los laboratorios así como la presencia de intereses e interpretaciones divergentes acerca de una realidad política y económica variable, dificultaron la generación de consensos que permitieran avanzar en el proyecto. En definitiva, el único laboratorio que construyó su planta está integrado a uno de los grupos más concentrados del sector, desenlace que muestra la profundización de las asimetrías entre empresas y empresarios en el marco de los procesos de neoliberalización.

Sin embargo, no puede perderse de vista un aspecto central del proceso de desarrollo del sur de la ciudad, que es la privatización de terrenos de origen público en favor de capitales privados, expulsando otros usos posibles. Y aquí ocurre como en el cuento del hortelano: las empresas acaparan terrenos en donde no concretan sus proyectos y que tampoco pueden ser utilizados para otras finalidades prioritarias teniendo en cuenta las necesidades de la población de las áreas en donde se emplazan. En este proceso, los empresarios ganan, pues acceden a la propiedad privada de terrenos urbanos en zonas desvalorizadas -a veces, como en este caso, con el apoyo directo o indirecto del Estado-, que retienen a la espera de oportunidades favorables para realizar operaciones mercantiles.

Todo esto refuerza y abre algunos de los interrogantes. Sin duda, el proyecto de desarrollo del sur se apoya en un proceso de mercantilización y se despliega -o pretende hacerlo- a través de alianzas público- 
privadas, pero también se observan una serie de debates y miradas contrapuestas al interior del Estado, así como una trama de resistencias que va penetrando en los requicios de la política e incide parcialmente en ella. Entonces ¿qué geografías sociales, políticas, urbanas van delinéandose en esta dinámica? En principio, pareciera que se trata de un mosaico irregular, con contornos más bien difusos. Por otra parte, teniendo en cuenta la volatilidad de las estrategias de los empresarios y las contradicciones que estos procesos producen al interior del Estado, interesa preguntarse cómo evolucionan estas áreas y qué implicancias tiene la acaparación de terrenos en ese contexto. Y, finalmente, no deja de ser interesante indagar en qué medida estos procesos inciden en la concentración de los sectores económicos involucrados.

\section{BiBliografía}

Abrutzky, R., Bramuglia, C. y Godio, C. (2015). El perfil de la industria farmacéutica de la Argentina. Interrogantes a mediano plazo. Ciencia, Docencia y Tecnología, 26 (51), 102-30

Arqueros Mejica, M. S. (2018a). Reflexiones en torno a la entramados público-privados: el caso del polo farmacéutico del sur de la ciudad de Buenos Aires. Cuaderno Urbano, 25 (25), 7-29.

Arqueros Mejica, M. S. (2018b). La política de desarrollo de la Comuna 8 de la ciudad de Buenos Aires (1996-2015) (Tesis de doctorado inédita). Universidad de Buenos Aires, Facultad de Ciencias Sociales, Ciudad Autónoma de Buenos Aires

Arqueros Mejica, M. S. y González Redondo, C. (2017). La política de distritos del sur la ciudad de Buenos Aires: una mirada en perspectiva. Revista Quid 16, [6], 7-29.

Atkinson, P. \& Coffey, A. (2011). Analysing documentary realities. En: Silverman, D. Qualitative Research. Issues of theory, methods and practice. London: Sage.

Azpiazu, D. (1999) La industria farmacéutica. Las estructuras oligopólicas frente a la desregulación y la apertura de la economía. En Azpiazu, D., Gutman, G. y Vispo, A. (comps.), La desregulación de los mercados. Paradigmas e inequidades de las politicas del neoliberalismo. Buenos Aires: Grupo Editorial Norma/FLACSO.

Azpiazu, D. y Schorr, M. (2008). Del modelo de los noventa a la posconvertibilidad. Reflexiones preliminares. Realidad Económica, (240), 29-59.

Beltrán, G. (2011).Las paradojas de la acción empresaria. Las asociaciones del empresariado argentino y la persistencia de las reformas estructurales. En: Pucciarelli, A. (coord.), Los años de Menem. La construcción del orden neoliberal. Buenos Aires: Siglo XXI editores.

Bercovich, L. y Maurino, G. (2013). Los derechos sociales en el gran Buenos Aires. Buenos Aires: Eudeba

Brenner, N; Peck, J.; Theodore, N (2013). ¿Y después de la neoliberalización? Estrategias metodológicas para la investigación de las transformaciones regulatorias contemporáneas. Urban, [01], 21-40

Castellani, A. y Gaggero, A. (2011). Estado y grupos económicos en la Argentina de los noventa. En: Pucciarelli, A. (coord.), Los años de Menem. La construcción del orden neoliberal. Buenos Aires: Siglo XXI editores.

Chiara, M. (2012). ¿Restricciones o incentivos? Revisando las tensiones del contexto desde una caracterización del régimen de implementación. En: Chiara, M. (coord.), Salud, politica y territorio en el Gran Buenos Aires. Los Polvorines: UNGS.

Cochrane, A. (1999). Redefining urban politics for the thwenty-first century. En: Andrew, E. G. \& Jonas, W. (eds.), The urban growth machine. Critical perspectives two decades later. New York: State University of New York Press.

Cosacov, N. (2014). Usos del suelo y judicialización en Buenos Aires. Las protestas contra la construcción de nuevos edificios en el barrio de Caballito. En: Azuela, A. y Cancino, M. A. (coords.), Jueces y conflictos urbanos en América Latina. México: Procuraduría Ambiental y de Ordenamiento Territorial Federal.

Dossi, M. y Lissin, L. (2011). La acción empresarial organizada: propuesta de abordaje para el estudio del empresariado. Revista Mexicana de Sociología, 73(3), 415-443.

Féliz. M. y López, E. (2012). Proyecto neodesarrollista en Argentina ¿modelo nacional-popular o nueva etapa en el desarrollo capitalista? La Plata: Editorial El Colectivo/Herramienta. 
Goicoechea, M. E. (2016). Distritos creatives en el sur de la ciudad de Buenos Aires (2008-2015). Renovación urbana y nuevas lógicas de segregación (Tesis de doctorado inédita). Universidad de Buenos Aires, Facultad de Ciencias Sociales, Ciudad Autónoma de Buenos Aires.

Harvey, D. (2007). Espacios del capital. Hacia una geografía crítica. Madrid: Akal.

Harvey, D. (2005). Breve historia del neoliberalismo. Madrid: Akal.

\section{Fuentes:}

INDEC (2001).Censo Nacional de Población, Hogares y Viviendas . Disponible en: https://www.indec.gob.ar/micro _sitios/webcenso/provincias_2/provincias.asp

INDEC (2010) Censo Nacional de Población, Hogares y Viviendas. Disponible en: https://redatam.indec.gob.ar/argbin/RpWebEngine.exe/PortalAction?\&MODE=MAIN\&BASE=CPV2 010B\&MAIN=WebServerMain.inl\&_ga=2.69178389.541829704.1586311865-9777977719.1586311865

Kowarick, L. (1996). Expoliación urbana, luchas sociales y ciudadanía: retazos de nuestra historia reciente. Estudios Sociológicos, 14 (42), 729-743

Martin, D. (2011). Urban politics as sociospatial struggles. InternationalJournal of Urban and Regional Research, 35 (4), $856-858$

Marques, E. (2016). De volta aos capitais para melhor entender as políticas urbanas. Novos Estudos CEBRAP, 35 [02], $15-33$

Melé, P. (2016). ¿Qué producen los conflictos urbanos?. En: Carrión, F. y Erazo, J. (coords.), El derecho a la ciudad en América Latina. Visiones desde la política. Ciudad de México: UNAM.

Merlinsky, G. (2016). "Efectos de las causas estructurales en el largo plazo: la causa Riachuelo. Revista Direito e Praxis, $7(2), 397-420$

Oszlak, O. y O’Donnell, G. (2008 [1976]). Estado y políticas estatales en América Latina: hacia una estrategia de investigación. En: Acuña, C. (comp.), Lecturas sobre el Estado y las políticas públicas: retomando el debate de ayer para fortalecer el actual. Buenos Aires: Jefatura de Gabinete de Ministros de la Nación

Pírez, P. (1995). Actores sociales y gestión de la ciudad. Revista Ciudades, 7[(8), 1-15.

Rodríguez, M. C. (2008). Autogestión, politicas de hábitat y transformación social. Buenos Aires: Espacio Editorial

Rodríguez, M. C. y Di Virgilio, M. (2011).Coordenadas para el análisis de las políticas urbanas: un enfoque territorial. En: Rodriguez, M. C. y Di Virgilio, M. (comps.), Caleidoscopio de las politicas territoriales. Un rompecabezas para armar. Buenos Aires: Prometeo.

Rougieur, M. (2014). Reflexiones sobre la historia de la industria y las empresas en América Latina. Revista de Historia Industrial, (53), 13-23.

Sabatini, F. (1999). Conflictos ambientales y desarrollo sustentable en las regiones urbanas. Revista EURE, 22(68), 77-91.

Sanfelici, D. (2017). Um olhar critic sobre as repercussoes urbanas das políticas de distritos criativos. Quid 16, (16), $62-66$

Socoloff, I. (2013). Polos, distritos y enclaves en Buenos Aires. De la pedagogía del "inversor" a la inflación de los precios del suelo. En:Marin, J. (comp.), La ciudad empresa. Buenos Aires: Centro Cultural de la Cooperación.

Svampa, M. (2011). Extractivismo neodesarrollista, Gobiernos y Movimientos Sociales en América Latina. Problèmes de l'Amérique Latine, (81), 103-128.

Theodore, N; Peck, J.; Brenner, N. (2009). “Urbanismo neoliberal: la ciudad y el imperido de los mercados”. Temas Sociales, (66), 1-11.

GCBA (2004a). Programa General de Acción de Gobierno 2005-2007. Buenos Aires: Gobierno de la Ciudad de Buenos Aires. 
GCBA (2004b). Un nuevo impulso para el desarrollo del sur de la ciudad. Publicado en el portal de noticias del GCBA. Disponible en: http://www.bsas.gov.ar/noticias/noticias/?modulo=ver\&item_id=10334\&contenido_id=333 \&idioma $=e s$

GCBA-IVC (2016). Informe final censo Barrio 20. Buenos Aires: Instituto de la Vivienda de la Ciudad. Disponible en: http://www.buenosaires.gob.ar/sites/gcaba/files/informe_censo_barrio_20_final.pdf

GCBA-Subsecretaría de Atención Ciudadana (2010). Audiencia Pública: Análisis del proyecto de Polo Farmacéutico, sito en las avenidas Escala y Fernández de la Cruz. Versión Taquigráfica. Buenos Aires: Jefatura de Gabinete del Gobierno de la Ciudad Autónoma de Buenos Aires.

Juncal, S.; et. alt. (2013). Análisis de diagnóstico tecnológico sectorial. Farmacéutico. Buenos Aires: Ministerio de Ciencia, Tecnología e Innovación Productiva-Presidencia de la Nación.

Legislatura Porteña (2005a). Acta de 12a Sesión Ordinaria - 26 de mayo de 2005. Versión Taquigráfica. Buenos Aires: Dirección General de Taquígrafos-Legislatura de la Ciudad Autónoma de Buenos Aires.

Legislatura Porteña (2005b). Acta de $1^{a}$ Sesión Especial - 26 de mayo de 2005. Versión Taquigráfica. Buenos Aires: Dirección General de Taquígrafos-Legislatura de la Ciudad Autónoma de Buenos Aires.

Legislatura Porteña (2004a). Acta de la 33a Sesión Ordinaria - 28 de octubre de 2004. Versión Taquigráfica. Buenos Aires: Dirección General de Taquígrafos-Legislatura de la Ciudad Autónoma de Buenos Aires.

Legislatura Porteña (2004b). Audiencia Pública:Incorporación del uso 'Polo Tecnológico' al Código de Planeamiento Urbano. Versión taquigráfica. Buenos Aires: Dirección General de Taquígrafos-Legislatura de la Ciudad Autónoma de Buenos Aires. Disponible en: https://www.legislatura.gov.ar/seccion/versiones-taquigraficas.ht $\mathrm{ml}$

Poder Judicial de la Ciudad Autónoma de Buenos Aires (2005). Expediente judicial 16693/0, caratulado "JUAREZ SARA ESTEL CONTRA GCBA SOBRE AMPARO (ART. 14 CABA)". Buenos Aires.

Redacción Clarín (2004).Ibarra anunció la creación de un polo farmacéutico en Soldati. Clarín, 06/10/2004. Buenos Aires

Redacción La Nación (2004). Piden rapidez para el Polo Farmacéutico. La Nación 11/10/2004. Buenos Aires.

Redacción La Razón (2004). Iniciativa del gobierno porteño y el sector privado. Proyectan en Villa Soldati el primer Polo Farmacéutico de la Ciudad. La Razón 24/05/2004. Buenos Aires.

\section{Notas}

* Doctora en Ciencias Sociales de la Universidad de Buenos Aires, Magíster en Economía Urbana de la Universidad Torcuato Di Tella y Licenciada en Sociología de la Universidad de Buenos Aires. En la actualidad se desempeña como becaria posdoctral del CONICET con sede en el Instituto de Investigaciones Gino Germani. Es JTP regular de la Universidad Nacional de Avellaneda, profesora en la Maestría en Hábitat y Pobreza Urbana de la Facultad de Arquitectura, Diseño y Urbanismo de la Universidad de Buenos Aires y de la Maestría en Desarrollo Territorial y Urbano co-gestionada por la Universidad Nacional de Quilmes y la Universidad Nacional de Avellaneda.

1 A partir del año 2008 la política de distritos tomó particular fuerza en la estrategia del desarrollo del sur de la ciudad de Buenos Aires (Goicoechea, 2016). En la actualidad, en esta zona se emplazan cuatro distritos (de las Artes, del Diseño, Tecnológico y del Deporte), en donde el ejecutivo local promueve la radicación de industrias y actividades de un mismo sector económico. Para ello, el GCBA ha desarrollado diversas obras de infraestructura urbana y de transporte y otorgado una serie de beneficios y exenciones impositivas a las empresas interesadas (Arqueros Mejica y González Redondo, 2017). $\mathrm{Al}$ igual que en el caso del Polo Farmacéutico, esta política enfatiza la capacidad de las articulaciones público-privadas como motor del "desarrollo".

2 De acuerdo a los datos del Censo Nacional de Población, Hogares y Viviendas de 2010, el 13,8\% de los hogares de esta área tienen Necesidades Básicas Insatisfechas, valor que está muy por encima del 7\% registrado a escala de la ciudad. Lo mismo ocurre con el déficit habitacional, que en la Comuna 8 se ubicó en torno al 8,3\% y en la ciudad al 5\%. El hacinamiento total alcanzaba al 23,3\% de los hogares de la Comuna 8 (el 5,1\% estaba en situación de hacinamiento crítico y el $18,2 \%$ no crítico) mientras que en la ciudad alcanzaba al 10,1\% de los hogares. Por último, es interesante considerar la variación intercensal entre los años 2001 y 2010, que en la ciudad fue del 3,9\%, en la Comuna 8 del 13,7\% 
y en las villas de esa comuna del 26,2\% (Arqueros Mejica, 2018). Esto evidencia que las villas tuvieron un incremento poblacional más dinámico que la Comuna 8 y que la ciudad en el último periodo intercensal.

3 Esta conceptualización conlleva implícita una mirada soble el Estado. Por un lado, se retomaron una serie de estudios que lo entienden como el componente específicamente político de la dominación y que ponen de relieve su naturaleza compleja y contradictoria (García Linera, 2010; Thwaites Rey, 2005; O’Donnell, 1977). La comprensión de que su principal tarea es garantizar la relación social capitalista, permite dar cuenta de su autonomía relativa con respecto a las clases sociales y, al mismo tiempo, del estrecho vínculo que existe entre éste y la sociedad y entre política y economía (García Linera, 2010; Thwaites Rey, 2005).

4 Como señalan Castellani y Gaggero (2011), la convertibilidad Argentina afectó a varios sectores de la economía, que durante este periodo tendió a extranjerizarse. Uno de esos sectores fue el famacéutico, en donde el aumento de la participación de los laboratorios extranjeros (Azpiazu, 1999) dio lugar a una mayor concentración de la industria y generó asimetrías de poder en el mercado interno (Abrutzky, et. al., 2015).

5 La Corporación Buenos Aires Sur fue creada en el año 2000 a través de la ley 470 y constituye la primera cristalización institucional para desarrollar el sur de la ciudad. Esta agencia de desarrollo fue pensada originalmente como un organismo gubernativo-gerencial capaz de dinamizar la gestión de proyectos, sortenado las rigideces de las burocracias preexistentes y los mecanismos institucionalizados. Aunque en sus primeros años mantuvo una mirada planificadora, hacia fines del año 2003 viró hacia una lógica de intervención fragmentaria en términos territoriales, que priorizó la dimensión económica del desarrollo y la participación del capital privado en la definición de acciones.

6 La Villa 20, como otros barrios de emergencia de la ciudad, se originó en la década de 1940 en el contexto de las migraciones rural-urbanas, a partir de la ocupación de terrenos vacantes por parte de familias sin posibilidad de acceder a la vivienda a través del mercado o del Estado. Se trata de un barrio autoconstruido, en donde el acceso a la infraestructura de servicios básicos y de saneamiento es deficitaria, y que presenta carencias de equipamiento e infraestructura urbana. De acuerdo a datos aportados por el IVC (GCBA-IVC, 2016), los habitantes de Villa 20 representaban en 2016 el $1 \%$ del total de la ciudad y el $15 \%$ de la Comuna 8.

7 Existe cierto consenso en la bibliografía en que a partir del año 2003 (y hasta el año 2015) se produjo un cambio a nivel regional en la estrategia de acumulación del capital, abriendo un periodo identificado como neodesarrollismo. En este contexto se produjeron cambios en las políticas macroeconómicas de nivel nacional, en el rol e injerencia del Estado y, en definitiva, en las relaciones entre el Estado, el mercado y la sociedad (Féliz y López, 2012; Azpiazu y Schorr, 2010; Svampa, 2011).

8 A mediados de la década de 1990 la cuestión del desarrollo del sur de la ciudad reingresó a la agenda estatal. En su problematización se identificaban dos perspectivas en competencia: la de desequilibrios territoriales norte-sur, y la de desigualdades entre ambas áreas de la ciudad. En esa discusión se debatía si la política urbana tenía que apuntar a extender las formas de desarrollo del norte (en particular lo que refiere a la dinamización del mercado del suelo) al sur; o bien, resolver las necesidades socio-económicas de la población de la zona sur, respetando su perfil, identidad e impronta (Arqueros Mejica, 2018b).

9 El barrio General Savio (Lugano I y II) comenzó a construirse en la década de 1960 durante la dictadura del General Onganía, en el marco del Plan Piloto del Parque Almirante Brown. Las condiciones de habitabilidad de este conjunto habitacional son precarias y buena parte de sus habitantes aun carecen de las escrituras de dominio de sus viviendas.

10 Posteriormente, a la resistencia encabezada por la Coordinadora, se sumaron nuevos grupos, como el Movimiento de Trabajadores Desocupados de Villa 20 y la Unión de Trabajadores Desocupados.

11 Esto se inscribe en un accionar más amplio del poder judicial, que en este periodo sobrepuso sus competencias al ejecutivo en materia de política pública. Algunos ejemplos son el fallo Mendoza sobre la Cuenca Matanza- Riachuelo (Merlinsky, 2016) y los diversos fallos en relación a las elecciones y urbanización de villas (Bercovich y Maurino, 2013).

12 Un dato de color es que uno de los diputados propuso incluir un artículo para que el $20 \%$ de los puestos de trabajo del Polo fueran ocupados por habitantes del área. La propuesta fue rechazada.

13 El Distrito Tecnológico de Parque Patricios fue creado en el año 2008 en el barrio de Parque Patricios, localizado también en el sur de la Ciudad de Buenos Aires. Este distrito está orientado a la promoción de la industria del software y tiene 190 empresas radicadas en su jurisdicción. Su emplazamiento ha producido un alza en los precios del suelo del barrio y estimulado un proceso de renovación de la zona (Goicoehcea, 2016).

14 El Centro de Atención al Inversor fue creado en el año 2007 en la estructura de la Subsecretaría de Inversiones del Gobierno de la Ciudad, siguiendo el modelo de las Agencias de Promoción de Inversiones (APIS). Esta dependencia tuvo entre sus principales funciones la relación con empresarios interesados en desarrollar negocios en y con el espacio urbano en la Ciudad de Buenos Aires.

15 En este sentido, a partir del año 2007 el Estado Nacional comenzó a regular los precios de los medicamentos. Según los laboratorios, esto incidió en su rentabilidad, sobre todo en el caso de los más pequeños, debido a que su producción depende de la compra de insumos activos en el mercado internacional. 
María Soledad Arqueros Mejica*. Políticas urbanas, conflicto y territorio: complejidades de la acc...

\section{BY-NC-SA}

